\title{
ANALYSIS OF USE CASES WHERE SECONDARY NOTATION COULD IMPROVE THE QUALITY OF CONCEPTUAL MODELLING
}

\author{
Ana-Maria GHIRAN \\ Business Informatics Research Center, Babeș-Bolyai University, Cluj-Napoca \\ anamaria.ghiran@econ.ubbcluj.ro
}

\begin{abstract}
Business process models play an important role in facilitating communication between various stakeholders as they can give an overview of the activities from the organization. For this purpose, business process models should be created in a way to best convey meaning. The quality of the models is greatly influenced by the presentation format of diagrammatic representation. Secondary notation is what the modeller can add on top of the formal syntax to emphasize certain information. However, the modellers might not be aware of the impact of the secondary notation and using it without a clear understanding about its role, e.g. for ornamental purposes could even have a negative effect on the transmitted knowledge. Therefore, we promote the idea that secondary notation should be suggested by the modelling environment based on an analysis of the context semantics. This paper presents an investigation over the role of secondary notation and it also skims the literature for use cases where secondary notation has proven to improve the expressivity of the created models.
\end{abstract}

Keywords: conceptual modelling, quality of models, understandability of models, secondary notation

JEL classification: D83, C63

DOI: $10.12948 / \mathrm{ie} 2019.04 .14$

\section{Introduction}

The graphical modelling of business processes has gained increased popularity and correspondingly many tools emerged, e.g. Bizzagi, Adonis, Bee-up etc. or modelling languages e.g. BPMN, UML Diagrams, Data flow diagrams, Petri Nets etc. Business operations are crucial to organizations and their "understanding is a prerequisite to conduct process analysis, redesign or execution" [1], therefore it is essential to have a description of the various business concepts' structures and data relationships. Conceptual modelling, as a general area that encompasses all kinds of representations of a system is more appropriate to consider, rather than focusing only on business process modelling.

Conceptual models make use of visual notations and how these are employed is influencing the quality of the models. As a result, for their evaluation some frameworks have been proposed [2], [3]. Among these, Moody's theory of "Physics of Notations" [2] is of particular interest for our investigation as it especially considers visual characteristics of a notation. We follow this research direction initially set by [4] and distinguish between primary and secondary notation. Primary notation given to a symbol refers to the formal definition offered by a modelling notation that corresponds to an adopted semantic and secondary notation refers to any graphical variation used in addition to the primary notation to emphasize or to clarify the meaning. That means secondary notation is usually applied at the discretion of the modeller. In some cases, the modeller can better convey meaning through it but in other cases it can conceal the intended message. An analysis of the implications produced by secondary notation should be considered as it could avoid communicating misunderstandings and facilitate transmitting a 
correct interpretation. But this is an open challenge known as design rationale for visual notations [5], as little attempts are made from the modellers side to justify the chosen symbols. This paper aims to investigate if such an evaluation is performed by the modellers before changing the notation and if they apply secondary notation because they are aware of its effects. In conducting our investigation, we systematically looked for use cases that covered the factors influencing the quality of the models, presumably that secondary notation should be employed with the goal to improve the quality of the models and not for ornamental purposes.

The work presented in this paper offers a starting point for identifying the elements that guide the modellers in applying secondary notation. This could help us formalize secondary notation in order to enable designing it in the modelling environment. This way, secondary notation is suggested by the tool rather than having the user decide over it and could guide the user in correctly adding it. The idea was put forward in [6] where we presented some techniques to enable secondary notation in conceptual models driven by semantics.

The remainder of the paper is organized as follows: section 2 presents background information on modelling language notation and particularly on secondary notation, then section 3 discusses model quality factors and various use cases that deal with secondary notation in conceptual modelling and last section includes concluding remarks.

\section{Background}

The notation refers to the set of symbols that visually represent the concepts and constitutes one of the main building blocks of a modelling language beside syntax (the rules for creating models or the grammar) and semantics (the consented meaning) [7]. Designing these artefacts (hence including notation) depends on the type of the modelling language, i.e. if there is a standardized language for which a common agreement in a large community must be obtained (like UML, BPMN, etc) of whether it is a domain specific modelling language that employs a metamodeling approach (like ADOxx [8]) to adapt it according to the specificity of the business situational requirements in the sense described by [7]. In the first case, there is little flexibility regarding the modifications of the notations as opposed to the last case that enables complete customization.

The primary notation would refer to the visual representation for a concept that is set at the metamodel level. Modellers can employ different visual appearance for the same process (given by colour, size, layout) and can still keep its logical structure and semantic interpretation. This variation permitted by some implementation tools to have or change visual variables is secondary notation [9]. Unintentional variation or an inconsistency use of the visual variables can alter the intended meaning and is referred as visual noise [2], which should be avoided.

Petre [4] emphasized that secondary notation should be carefully applied to obtain cognitive effective visual notations. This means they are optimized for processing by human mind in order to enable a quick and exact understanding of the diagram. Kahneman's highly influential work on cognitive psychology [10] pointed out over two types of processing: one that is fast, instinctive, emotional and automatic and the other one that is slower, subject to conscious judgements and previous knowledge. The first one occurs straight away when we see the picture while the other one occurs at a second stage when we are trying to comprehend the information transmitted in the diagram. If the processing burden from the second phase could be shifted towards the first one, we get what is called in cognitive psychology "computational offloading". This is the explanation why it is easier for us to perceive pictures instead of text: following a sequence of characters and decoding the words requires greater cognitive effort [2]. 
This revelation is embraced here as the main purpose for secondary notation: to obtain cognitive offloading. More precisely, this must be used to overcome [11]: capacity limitations (like the amount of information that is active in our working memory or that we can accurately perceive only a relatively small region of our visual field), computational effort (integration with prior knowledge).

\section{Secondary notation use cases}

To systematically approach the literature review for use cases of secondary notation in conceptual modelling, we need to proceed in an organized way that would enable to fully address the area. For this, we devise a taxonomy that covers the factors influencing the quality of the models.

In conceptual modelling, quality of the models has been an important topic for research and many frameworks have been proposed to evaluate it: CMQF - Conceptual Modelling Quality Framework [3] SEQUAL [12], 7BPM [13]. A common and major attribute in all these frameworks is the model's pragmatic quality, which is the correspondence between the model on one side and the interpretation given to it on the other side [12]. Therefore, the pragmatic quality is closely linked to understandability of the models which can be defined as the effort required to read and correctly interpret the model content [5].

We can broadly divide the factors influencing the understandability of the models in two large categories: internal stimulus and external support. Each of them is further classified into more detailed factors and for each one we identified studies that deal with it.

Table 1. Factors influencing the understandability of the models

\begin{tabular}{|c|c|c|}
\hline \multicolumn{2}{|c|}{ Factors influencing the quality of models } & Use cases for Secondary Notation \\
\hline \multicolumn{3}{|c|}{ Internal } \\
\hline $\begin{array}{c}\text { Personal } \\
\text { Characteristics }\end{array}$ & $\begin{array}{ll}- & \text { experience with conceptual } \\
\text { modelling } \\
\text { - } & \text { familiarity with the domain } \\
\text { - } & \text { personal style }\end{array}$ & $\begin{array}{l}\text { using guidelines and recommendations in } \\
\text { notations }\end{array}$ \\
\hline \multicolumn{3}{|l|}{ External } \\
\hline $\begin{array}{l}\text { Modelling } \\
\text { language }\end{array}$ & $\begin{array}{ll}\text { - } & \text { number of formal concepts } \\
\text { - } & \text { graphical representation of } \\
\text { the symbols }\end{array}$ & $\begin{array}{l}\text { - escape from formalism (express information } \\
\text { that is not found in that chosen language) by } \\
\text { using a metamodeling method } \\
\text { - enhance semantics from concept' appearance }\end{array}$ \\
\hline $\begin{array}{c}\text { Model } \\
\text { complexity }\end{array}$ & $\begin{array}{ll}- & \text { number of concepts } \\
- & \text { number of connections } \\
- & \text { the interactivity of model } \\
\text { elements } \\
\text { - } & \text { the flow pattern }\end{array}$ & $\begin{array}{ll}- & \text { emphasizing some elements } \\
\text { - } & \text { representing corresponding elements as an } \\
\text { ensemble (e.g. coloring similar concepts, } \\
\text { grouping etc.) } \\
\text { - impose structuredness (avoid crosslinking), }\end{array}$ \\
\hline
\end{tabular}

\subsection{Internal factors}

The quality of the models is highly affected by the personal characteristics of the individual that designs them. Here we can further consider as influence factors the competences in conceptual modelling, the familiarity with the domain or domain knowledge, personal style in creating the models and previous experience in modelling.

Modelling requires knowledge both a) in finding the right abstractions, correct usage of specializations and aggregations of concepts and b) in describing the domain. Often there is a tradeoff: modelling experts are missing a deeper understanding of the domain concepts and domain experts are not familiar with modelling notations [14].

Pinggera et al. [15] acknowledged that modellers can adopt a certain style in developing process models. They identified three types of styles: 1) "an efficient modelling style" - a fast 
addition of the process elements on the model constrained by time limits, 2) "a layout driven modelling style" devoting time in creating comprehensiveness of the model but being less efficient in creating the model and 3) "an intermediate modelling style" - as a hybrid between the previous two styles.

Regarding this category of factors most of the studies were concerned with the analysis of the comprehensibility of the models from the point of view of the person interpreting the models [16][17][18]. We assume that the lack of studies regarding the influence of the modellers' expertise on the models' quality is determined by the clear intuition that the experience has a profound impact.

However, authors in [19] are studying the effect of the expertise, showing that creating process models using swimlanes will enable novice users to correctly understand the domain. A conclusion from this is that using expertise on the encoding side (when creating models) will decrease the level of the required knowledge on the decoding side (when reading the models). Another study [20] showed that how the names of the labels are formulated is having a certain influence on the interpretation of the models. Again, this skill is acquired by practice.

Applying secondary notation is more likely to be encountered on modellers with expertise that are aware they should redirect the readers' attention on the relevant parts of the model.

\subsection{External factors}

The modelling activity can be supported or hindered by factors that are not related to the individual but are concerning the modelling language/tool or model complexity.

As shown by [18] the characteristics of the modelling language can have a direct impact on the comprehension of the models. Also [21] are pointing out that the language complexity can prevent the users in knowing all of them and as a solution, the modellers are restricting the number of used concepts by adopting only small subsets. For instance, in case of the BPMN standard, authors from [21] actually claim that less than $20 \%$ of its vocabulary is regularly used and some of its concepts are never employed in practice. Therefore, there are cases when some modellers will use a subset of the modelling language whilst others will use other subset, which implies small differences regarding the meaning that they would like to convey through their models.

On the other hand, modellers sometimes want to include concepts that are not available on the standardized modelling language and for which they will use substitutes [22].

Based on these findings we can state that both the number of the formal concepts included in the modelling language as well as the graphical representation of the symbols are factors that affect the understandability of the models.

Many researchers therefore have evaluated different modelling languages to asses which one is better in conveying meaning: for instance, [22] analysed symbol sets from various process modelling notations (EPC, UML Activity Diagrams, YAWL and BPMN) by checking their coherence with the principles stated by Moody (regarding the characteristics needed to be satisfied by the modelling notation).

As modellers use tool-specific implementations for standardized languages [23], further notational enhancements are possible to some extent, e.g. some tools allow changing some of the visual variables (color, texture, size etc.) to be applied to the model's concepts. There is also the situation when a standardized language can be imported in a metamodeling platform and readjust it if needed, as for example the Bee-up toolkit [24].

Researchers [17] showed that the more complex is a model both in terms of the number of elements and of the number of connections between them, the more difficult is to understand. This is explained using the Cognitive Load Theory [25] which assumes a limited processing capacity of the human working memory. 
www.conferenceie.ase.ro

Colouring corresponding elements [9] [26] has been proposed as one solution for dealing with the complexity of the models and generally grouping multiple objects sharing similar characteristics into an entity [27] is beneficial because it will reduce the number of the elements in the working memory load, hence increase the probability to effectively and efficiently understand the model.

The complexity of the model can also manifest in the presence of certain control flow patterns (such as conditional or parallel execution tasks in case of process models) or the interactivity of the model elements, which as well increases the cognitive difficulty of reasoning tasks [28] Handling the unstructured flow of a process model has been addressed by [29] who proposed a way to automatically transform process models with arbitrary topology into equivalent wellstructured models.

\section{Conclusions}

The comprehensibility of models has been actively studied either empirically, by designing some experiments to test the influences of some variables like model complexity, the language features, the personal characteristics of the modeller or theoretically, by relying on explicit scientific studies from psychology like cognitive load theory.

All factors do matter and one cannot state which are more important, but some are inherent, like size and content of the model, or the personal characteristics of the modeller, consequently these can hardly be modified. Notational aspects are also among the influencing factors of the understandability of the models, but in contrast they can be changed or in other cases some variability can added on the symbols.

Secondary notation represents one instrument that can enhance the communication value of the conceptual models. This paper advocates the idea that secondary notation should not be solely the initiative of the modeller but rather it should be induced by the modelling environment based on a semantic analysis of the concept and of its context.

\section{References}

[1] M. Dumas, M. La Rosa, J. Mendling, H. A. Reijers et al., "Fundamentals of business process management," vol. 1, Springer, 2013.

[2] D. Moody, "The "physics" of notations: toward a scientific basis for constructing visual notations in software engineering," IEEE Transactions on software engineering, vol. 35, no. 6, pp.756-779, 2009

[3] H. J. Nelson, G. Poels, M. Genero, M. Piattini, "A conceptual modeling quality framework," Software Quality Journal, 20(1), 201-22, 2012

[4] M. Petre, "Why looking isn't always seeing: readership skills and graphical programming," Communications of the ACM, vol. 38, no. 6, pp. 33-44, 1995

[5] D.L. Moody, G. Sindre, T. Brasethvik, A. Sølvberg, "Evaluating the quality of information models: empirical testing of a conceptual model quality framework," In Proceedings of the 25th international conference on software engineering (pp. 295-305). IEEE Computer Society, 2003

[6] A.M. Ghiran, R.A. Buchmann, D. Karagiannis, "Towards a framework of techniques for enabling semantics-driven secondary notation in conceptual models," In 2018 12th International Conference on Research Challenges in Information Science (RCIS) (pp. 1-6). IEEE, 2018

[7] D. Karagiannis and H. Kuhn, "Metamodelling platforms," in" EC-Web, vol. 2455, p. 182, 2002

[8] BOC GmbH. (2018, Apr.) The adoxx metamodelling platform. [Online]. Available: http://www.adoxx.org/live 
www.conferenceie.ase.ro

[9] H. A. Reijers, T. Freytag, J. Mendling, and A. Eckleder, "Syntax highlighting in business process models," Decision Support Systems, vol. 51, no. 3, pp. 339-349, 2011

[10] D. Kahneman, Thinking, Fast and Slow, Published by Farrar, Straus and Giroux, 2012

[11] E.F. Risko, and S.J. Gilbert, Cognitive offloading. Trends in Cognitive Sciences, 20(9), pp. 676-688, 2016

[12] J. Krogstie, "Quality in business process modeling," Springer; 2016.

[13] J. Mendling, H.A. Reijers, W.M. van der Aalst, "Seven process modeling guidelines (7PMG)," Information and Software Technology, vol. 52, no. 2, pp.127-136, 2010.

[14] D. Ionita, R. Wieringa, J.W. Bullee, and A. Vasenev, "Tangible modelling to elicit domain knowledge: an experiment and focus group," In International Conference on Conceptual Modeling (pp. 558-565). Springer, 2015

[15] J. Pinggera, P. Soffer, D. Fahland, M. Weidlich, S. Zugal, B. Weber, H. A. Reijers, and J. Mendling, "Styles in business process modeling: an exploration and a model," Software \& Systems Modeling, vol. 14, no. 3, pp. 1055-1080, 2015

[16] J. Mendling, M. Strembeck, J. Recker, "Factors of process model comprehension - findings from a series of experiments," Decision Support Systems, vol. 53, no. 1, pp.195-206, 2012

[17] H.A. Reijers, J. Mendling, "A study into the factors that influence the understandability of business process models," IEEE Transactions on Systems, Man, and Cybernetics-Part A: Systems and Humans, vol. 41, no. 3, pp.449-462, 2011

[18] J. Hahn and J. Kim, "Why are some diagrams easier to work with? effects of diagrammatic representation on the cognitive integration process of systems analysis and design," ACM Transactions on Computer Human Interaction (TOCHI), vol. 6, no. 3, pp. 181-213, 1999.

[19] P. Bera, "Does cognitive overload matter in understanding BPMN models?" Journal of Computer Information Systems, vol. 52, no. 4, pp.59-69, 2012

[20] J. Mendling, H.A. Reijers, J. Recker, "Activity labeling in process modeling: Empirical insights and recommendations," Information Systems, vol. 35, no. 4, pp.467-482, 2010

[21] M. Zur Muehlen and J. Recker, "How much language is enough? Theoretical and practical use of the business process modeling notation". In Seminal Contributions to Information Systems Engineering (pp. 429-443). Springer, Berlin, Heidelberg, 2013.

[22] K. Figl, J. Mendling, M. Strembeck, "The influence of notational deficiencies on process model comprehension," Journal of the Association for Information Systems, vol. 14, no. 6, p.1, 2013

[23] J. Stark, W. Esswein, "Using secondary notation to improve the cognitive effectiveness of BPMN-models," 2017

[24] OMiLAB. (2019, Apr.) Open models initiative laboratory, Bee-Up tool [Online]. Available: http://austria.omilab.org/psm/content/bee-up/info

[25] P.A. Kirschner, Cognitive load theory: Implications of cognitive load theory on the design of learning, 2002

[26] R. Petrusel, J. Mendling, H. A. Reijers, "Task-specific visual cues for improving process model understanding," Information and Software Technology, vol. 79, pp. 63-78, 2016

[27] G.A. Alvarez, "Representing multiple objects as an ensemble enhances visual cognition," Trends in cognitive sciences, vol. 15, no. 3, pp.122-131, 2011

[28] K. Figl, R. Laue, "Influence factors for local comprehensibility of process models," International Journal of Human-Computer Studies, vol. 82, pp.96-110, 2015

[29] A. Polyvyanyy, L. García-Bañuelos, M. Dumas, "Structuring acyclic process models," In International Conference on Business Process Management (pp. 276-293). Springer, Berlin, Heidelberg, 2010 